\title{
A fitting formula for the merger timescale of galaxies in hierarchical clustering
}

\author{
C. Y. Jiang ${ }^{1,2}$, Y. P. Jing ${ }^{1}$, A. Faltenbacher ${ }^{1}$, W. P. Lin ${ }^{1}$, Cheng $\operatorname{Li}^{1}$ \\ ${ }^{1}$ Shanghai Astronomical Observatory, Nandan Road 80, Shanghai, China \\ ${ }^{2}$ Graduate School of the Chinese Academy of Sciences, 19A, Yuquan Road, Beijing, China
}

\begin{abstract}
We study galaxy mergers using a high-resolution cosmological hydro/N-body simulation with star formation, and compare the measured merger timescales with theoretical predictions based on the Chandrasekhar formula. In contrast to Navarro et al., our numerical results indicate, that the commonly used equation for the merger timescale given by Lacey and Cole, systematically underestimates the merger timescales for minor mergers and overestimates those for major mergers. This behavior is partly explained by the poor performance of their expression for the Coulomb logarithm, $\ln \left(m_{\text {pri }} / m_{\text {sat }}\right)$. The two alternative forms $\ln \left(1+m_{\text {pri }} / m_{\text {sat }}\right)$ and $1 / 2 \ln \left[1+\left(m_{\text {pri }} / m_{\text {sat }}\right)^{2}\right]$ for the Coulomb logarithm can account for the mass dependence of merger timescale successfully, but both of them underestimate the merger time scale by a factor 2 . Since $\ln \left(1+m_{\text {pri }} / m_{\text {sat }}\right)$ represents the mass dependence slightly better we adopt this expression for the Coulomb logarithm. Furthermore, we find that the dependence of the merger timescale on the circularity parameter $\epsilon$ is much weaker than the widely adopted power-law $\epsilon^{0.78}$, whereas $0.94 \epsilon^{0.60}+0.60$ provides a good match to the data. Based on these findings, we present an accurate and convenient fitting formula for the merger timescale of galaxies in cold dark matter models.
\end{abstract}

Subject headings: dark matter — galaxies: clusters: general — galaxies: kinematics and dynamics methods: numerical

\section{Introduction}

Dynamical friction plays a crucial role in the formation and evolution of galaxies. During the merger of two dark matter halos, galaxies in a less massive halo will become the satellite galaxies of the more massive one. These satellite galaxies gradually lose their energy and angular momentum under the action of dynamical friction and are predestined to sink to the center of the massive dark matter halo, if they are not disrupted by the tidal force.

Dynamical friction takes effect through interaction of galaxies with background dark matter particles. Chandrasekhar (1943) gave a description for this phenomenon for an idealized case where a rigid object moves through a uniform sea of collisionless matter particles. This description can be applied to the case of a satellite galaxy mov- ing in a dark matter halo. The orbits of dark matter are deflected by the galaxy, which produces an enhancement of dark matter density behind the galaxy. Consequently, the galaxy suffers a steady deceleration by the drag of the wake, and will eventually merge to the central galaxy of the dark matter halo. The merger timescale, i.e. the time elapsing between entering the virial radius of the dark matter halo and final coalescence of satellite and central galaxy, can be derived using Chandrasekhar's formula (see, e.g., Binney \& Tremaine 1987). Additionally, taking into account the dependence on the orbital circularity Lacey \& Cole (1993) derived the following expression for the merger timescale of a satellite galaxy orbiting around a massive halo with circular velocity $V_{\mathrm{c}}$

$$
T_{\text {Chandra }}=\frac{1}{2} \frac{f(\epsilon) V_{\mathrm{c}} r_{\mathrm{c}}^{2}}{C G m_{\mathrm{sat}} \ln \Lambda},
$$


where $\epsilon$ is the circularity parameter of the satellite's orbit and $r_{\mathrm{c}}$ is the radius of a circular orbit with the same energy as the satellite's orbit. $f(\epsilon)$ describes the dependence of $T_{\text {Chandra }}$ on the orbital circularity, and is approximated by $f(\epsilon) \sim \epsilon^{0.78}$ for $\epsilon>0.02$ (Lacey \& Cole 1993). $C$ is a constant, approximately equal to 0.43 , and $m_{\text {sat }}$ is the satellite mass. $\ln \Lambda$ is the Coulomb logarithm, which is given $\ln \left(d_{\max } / d_{\min }\right)$, where $d_{\max }$ is the maximum relevant impact parameter at which background particles are scattered into the wake and $d_{\min }$ is the minimum impact parameter (Chandrasekhar 1943; White 1976). It is expected to be applicable for cases where the satellite mass is much smaller than that of the primary halo.

There have been many works which used Nbody simulations to check the validity of Chandrasekhar's formula and its application to the merging of satellite and central galaxies, but no consensus has been reached on the accuracy of such applications. This is because a galaxy merger is a more complicated process than a pure motion of a rigid body through an uniform collisionless matter distribution as considered by Chandrasekhar. The primary halo has a density increasing inward to the halo center, which makes it nontrivial to choose the maximum impact parameter for the Coulomb logarithm (Hashimoto et al. 2003; Jiang \& Binney 2000; van den Bosch et al. 1999). Because the satellites lose their mass due to the tidal interaction by the primary halo, one has to follow both the trajectory and the mass evolution of the satellites to derive their merger timescale. Unfortunately, there is still a considerable amount of uncertainties in modeling these processes (Tormen et al. 1998; Gao et al. 2004; Zentner et al. 2005). A further complication is that due to the similar orbits of the tidally stripped mass and the satellite itself the tidal debris will trail the satellite for a significant amount of time which in turn will exert a drag force on the satellite (Fuiii et al. 2006; Fellhauer \& Lin 2007). Besides, the merger can alter the structure of the primary halo which is another complication for accurately computing the merger timescale (Zaritsky \& White 1988; Cora et al. 1997).

It is however very useful to give a simple prescription for the merger timescale of the satellites. Navarro et al. (1995) used an N- body/hydrodynamics simulation with gas cooling to determine the merger time scales. Their simulation didn't include a recipe for star formation, thus they used the cold gas at the cores of dark matter halos as a proxy for galaxies. They found a good agreement with the prediction of equation (1) if the satellite mass $m_{\text {sat }}$ is taken to be the sum of the cold gas core and the associated dark matter halo at the moment when it crosses the virial radius of the primary halo for the first time. They further pointed out that the predicted merger timescale is too long if only the cold gas is taken for the satellite mass.

The N-body study of Navarro et al. provides a strong support for using equation (1) to determine the merger timescale in both theoretical and observational studies, if $m_{\text {sat }}$ is taken to be the total mass of the satellite at the virial radius of the primary halo. For example, this equation is an important ingredient in modeling mergers of galaxies in analytical studies of galaxy formation (e.g., Kauffmann et al. 1999; Cole et al. 2000; Monaco et al. 2000; Somerville \& Primack 1999; Menci et al. 2002; Nagashima et al. 2002; Hatton et al. 2003; Khochfar \& Ostriker 2007; Baugh 2006, for an excellent review) and in understanding the merger rates of galaxies in the cosmological context (e.g. Ostriker \& Turner 1979; Lin et al. 2004; Gill et al. 2005; Maller et al. 2006; Conroy et al. 2007; White et al. 2007; Zheng et al. 2007). However, there are indications that the Navarro et al. prescription underestimates the merger time or overestimates the merger rate. Springel et al. (2001) and Kang et al. (2005) found that the luminosity of central galaxies in rich clusters is reduced if the orbital evolution of satellites is determined by high-resolution N-body simulations compared to the luminosities based on the Navarro et al. merger rates. We also note that the N-body experiment by Colpi et al. (1999) gave a merger timescale which is longer than what Navarro et al. suggests. They even found an much weaker dependence on the circularity with the exponent only about 0.4 (instead of 0.78 ). Therefore it is not yet clear what causes these discrepancies, especially the one between Colpi et al. and Navarro et al.. It would be helpful to point out that Navarro et al. used a cosmological hydro/N-body simulation with gas cooling and included both major and minor mergers in their 
study, while Colpi et al. used N-body simulations of galaxy mergers and considered minor mergers only.

In this paper, we will use a high-resolution hydro/N-body cosmological simulation to clarify this situation. In the simulation, gas cooling and star formation are included so that the galaxy mergers can be identified unambiguously and the merger timescale can be well measured. Our results can be directly compared with Navarro et al., therefore, they will be used to study the origin of the discrepancies mentioned above. We will show that the Navarro et al. prescription actually underestimates the merger time for minor merges, qualitatively in good agreement with Colpi et al., but overestimates it for major mergers. In light of our simulation results, we will propose an accurate fitting formula for the merger timescale that accounts well for the dependences on mass and circularity of the individual satellites, and can therefore accommodate both, minor and major merger events.

The paper is organized as follows. In Section 2 , we describe our simulation and our method for calculating the merger timescale in the simulation. Section 3 gives a comparison between our simulation result and the theoretical prediction. A new fitting formula for the merger timescale is derived in Section 4. Finally, we summarize our results in Section 5 .

\section{Merger timescales in simulation}

\subsection{The simulation}

A parallel version of the SPH code GADGET2 (Springel et al. 2001; Springel 2005) is used to simulate the structure formation and evolution in the Universe. The cosmological parameters we use are $\Omega_{\Lambda}=0.732, \Omega_{\mathrm{m}}=0.268, \Omega_{\mathrm{b}}=0.044, \sigma_{8}=0.85$, and a Hubble constant $H_{0}=100 h \mathrm{~km} \mathrm{~s}^{-1} \mathrm{Mpc}^{-1}$ with $h=0.71$. The box is $100 h^{-1} \mathrm{Mpc}$ on a side, with $512^{3}$ dark matter particles and $512^{3}$ gas particles. The resulting mass resolution for dark matter and gas particles is $4.6 \times 10^{8} h^{-1} \mathrm{M}_{\odot}$ and $9.2 \times 10^{7} h^{-1} \mathrm{M}_{\odot}$, respectively. The simulation includes the physical processes of radiative cooling and star formation. It also includes supernova feedback, outflows by galactic winds, and a sub-resolution multiphase model for the interstellar medium as detailed in Springel \& Hernquist
(2003). The simulation has the same mass resolution and model parameters as the star formation run of Jing et al. (2006), except that the softening length of the gravitational force is greatly reduced in the current simulation, where we use a spline kernel (Springel 2005), roughly equivalent to a Plummer force softening of $4.5 h^{-1} \mathrm{kpc}$ (comoving). There are a total of 88 snapshot outputs from $z=2.0$ to the present time, $z=0$, with an equal logarithmic scale factor interval of $\Delta \ln a=0.01$ between two consecutive outputs. The large number of outputs enables us to accurately sample orbits of satellites within massive halos. Both, the good force resolution and the dense sampling of snapshots, are crucial for the current study.

\subsection{Construction of halo merger trees}

Dark matter halos are identified using the friends-of-friends (FOF) method, with a linking length of 0.2 times the mean inter-particle separation. To obtain a sufficient number of halos with reasonable mass resolution, we only focus on the halos with masses $m_{\mathrm{vir}}>5 \times 10^{12} h^{-1} \mathrm{M}_{\odot}$ at the present epoch. The virial mass of a halo $m_{\text {vir }}$ is defined as the mass enclosed within the virial radius $r_{\text {vir }}$ within which the mean mass density is $\Delta(z)$ times the critical density of the universe at redshift z. For $\Delta(z)$ we adopt the fitting formula for flat universes provided by Bryan \& Norman (1998),

$$
\Delta(z)=18 \pi^{2}-82 x-39 x^{2},
$$

where $x$ is the density parameter for the vacuum density (the cosmological constant) at redshift $z$.

Then we trace these halos back to $z=2.0$ to construct the main branch of the merger tree for each halo. For halo $A$ at some snapshot, halo $B$ at an earlier snapshot which, among all its progenitors, contributes the largest number of particles to $A$ is defined as the main progenitor of $A$. All the other progenitors of halo $A$, each of which is required to have more than half its particles merging with $A$, are taken as satellite halos of halo $B$, while $B$ is called the primary halo. Note, we use 'satellite' to represent the whole halo, including both, dark and stellar matter.

We do not use the orbital energy as the criterion to identify a satellite as being bound or unbound, since an orbit that starts out unbound will 
not necessarily remain unbound, because dynamical friction may sufficiently reduce its energy, see e.g., Benson (2005). They find that only about 2\% of all initially unbound orbits fail to become bound and so escape from their primary halo. Furthermore, to reduce artificial effects caused by the finite numerical resolution we keep only those satellites that have central galaxies more massive than $2.0 \times 10^{10} h^{-1} \mathrm{M}_{\odot}$. Typically, these satellite galaxies are surrounded by a dark matter halo comprising more than 1000 particles before entering the primary halo.

\subsection{Merging timescale of galaxies}

The galaxies are also identified with the friendsof-friends method applied to the star particles but with a small linking length of $4.88 h^{-1} \mathrm{kpc}$. Besides the central galaxies of the primary halos we only focus on those galaxies which have been the central galaxies of the approaching satellite halos. Throughout, the former will be referred to as central and the latter as satellite galaxies. The descendant of a galaxy, $C$, is the galaxy in a subsequent snapshot which shares the most star particles with $C$. A galaxy merger is identified if the satellite galaxy and the central galaxy begin to have the same descendant at one snapshot, and continue to have the same descendant for the following four snapshots ( $\geq$ half of the dynamical time of a halo). We use this criterion to ensure that the merger is a real merger not just a close flyby.

The merger timescale is defined as the time elapsed between the moment when the satellite galaxy first crosses the virial radius of the primary halo and the final coalescence of satellite and central galaxy. The computation of the merger timescale from the simulation involves four distinct snapshots: snapshot $a$, the last snapshot for which the satellite halo is identified as a single halo; snapshots $b$ and $b+1$, between snapshot $b$ and snapshot $b+1$ the satellite galaxy crosses the virial radius of the primary halo for the first time; and finally, snapshot $c$, beginning of the coalescence of satellite and central galaxy. To accurately determine the point in time when the satellite galaxy enters the primary halo, we assume that the satellite galaxy moves with constant velocity from its actual location, both measured at snapshot $b$, until it hits the virial radius, which has been fixed at snapshot $a$. However, a substantial fraction $(\sim 14 \%)$ of the satellite galaxies do not reach the virial radius within the time interval between snapshot $b$ and $b+1$. This happens because, in general, satellites are in accelerated motion. In such cases, we choose snapshot $b+1$ as the time at which the satellite reaches the virial radius. Due to the dense time sampling by the large number of snapshots this uncertainty constitutes only a marginal source of error.

Finally, the merger timescale for each completed merger event is defined to be the interval between the time when the satellite first enters the virial radius and the middle point between snapshots $c$ and $c-1$.

Some basic statistical properties of the mergers are presented in Figure 1. There is almost an equal amount of major mergers and minor mergers, if we use the mass ratio $m_{\text {pri }} / m_{\text {sat }}=3$ as the dividing line. Since we examine only snapshots starting from redshift $z=2$, the redshifts, at which the eventually merging satellites first cross the virial radius of the primary halo, span the range between $z=0.4$ and $z=2$. (Satellites which approach more recently than $z=0.4$ do not have sufficient time to merge with the central galaxy.) The ratio of the stellar mass of a central galaxy to the dark matter mass of the primary halo varies from $0.5 \%$ to $5 \%$ with an average of $2 \%$. This ratio is in reasonable agreement with the observed values of galaxy groups Gonzalez et al. 2007; Lin et al. 2003). The satellite sample has a wide spectrum of orbital energies, as displayed by the distribution of $r_{c} / r_{\text {vir }}$, which ranges from 0.6 to 1.5 with an average 0.8 . Thus, we believe that our sample represents a typical sample of galaxy mergers.

\section{Comparison with theory}

Equation (1) is only applicable for mergers with mass ratios $m_{\text {pri }} / m_{\text {sat }} \gg 1$, where $m_{\text {pri }}$ and $m_{\text {sat }}$ stand for the mass of the primary and the satellite halo, respectively (Binney \& Tremaine 1987). As mentioned above $\Lambda$ in the Coulomb logarithm $\ln \Lambda$ is defined as the ratio between maximal and the minimal impact parameters $\left(d_{\max } / d_{\min }\right)$ for which encounters between the satellite and the dark matter particles can be considered effective . 
An equivalent expression for $\Lambda$ is given by

$$
\Lambda \equiv \frac{d_{\mathrm{max}} V_{\mathrm{typ}}^{2}}{G\left(m_{\mathrm{sat}}+m_{\mathrm{dm}}\right)}=\frac{m_{\mathrm{pri}}}{m_{\mathrm{sat}}},
$$

where $V_{\text {typ }}$ and $m_{\mathrm{dm}}$ are the typical velocity and mass of background dark matter particles. The transition from the middle to the expression on the right hand side is obtained by setting $d_{\max }=$ $r_{\text {pri }}$ (the radius of the primary halo), $V_{\text {typ }} \approx V_{\text {pri }}$ (the circular velocity of the primary halo), and assuming $m_{\mathrm{dm}} \ll m_{\text {sat }}$.

Therefore, according to equation (11) a correct estimate of the satellite mass is pivotal for the determination of the dynamical friction timescale. A satellite orbiting in the potential well of the primary halo loses a large fraction of its initial mass due to the exposure to the global tidal field (e.g., Tormen et al. 1998; Gao et al. 2004; Shaw et al. 2007) and due to high-speed encounters with other satellites (e.g., Moore et al. 1996; Gnedin 2003). Based on a hydro/N-body simulation Navarro et al. (1995) investigated the dependence of the dynamical friction time scale on the the Coulomb logarithm $\ln \Lambda=\ln \left(m_{\text {pri }} / m_{\text {sat }}\right)$ by considering two extreme choices for $m_{\text {sat }}$ : (1) $m_{\text {sat }}$ was considered to be the total virial mass of the satellite before entering the primary halo, i.e. the sum of the gas (representative for the stellar component in their simulation) and the cold dark matter within the satellite's virial radius, (2) $m_{\text {sat }}$ only accounted for the cold gas associated with the satellite galaxy at the center of the approaching dark matter halo. They found when the total virial mass is chosen for $m_{\text {sat }}$ equation (11) gives a good prediction for the merger time scale although the scatter is very large. If only the cold gas is adopted for $m_{\text {sat }}$ equation (1) significantly overestimates the merger timescale because the cold gas mass is always much smaller than the virial mass. Based on this numerical investigation, equation (11) with the initial satellite virial mass for $m_{\text {sat }}$ is widely used in galaxy formation studies (e.g., Cole et al. 2000; Kauffmann et al. 1999; Kang et al. 2005). Here, we also follow this convention for $m_{\text {sat }}$.

With the present analysis we aim to examine the validity of equation (1) by means of a cosmological high resolution N-body/hydro simulation. Figure 2 compares the merger timescale $T_{\text {Chandra }}$ computed according to equation (1) with the merging time $T_{\text {simu }}$ measured in the simulation. The solid diagonal displays $T_{\text {Chandra }}=$ $T_{\text {simu }}$. The results indicate a qualitative agreement between the prediction of equation (1) and the time scales measured from the simulation. However, scatter between $T_{\text {Chandra }}$ and $T_{\text {simu }}$ is extremely large. To see whether the large scatter is caused by the failure of equation (11) for mass ratios $m_{\text {sat }} / m_{\text {pri }} \approx 1$ we plot the median value of $T_{\text {simu }} / T_{\text {Chandra }}$ as a function of $m_{\text {sat }} / m_{\text {pri }}$ in Figure 3 (the solid line). The figure clearly shows that the time ratio increases monotonically with decreasing mass ratios. That is, the time ratio is significantly smaller than 1 ( 0.55 for the mass ratio larger than 0.65 ) for the major mergers and approaches 4 for minor mergers (for the mass ratio smaller than 0.065 ). This implies that equation (1), which is expected to be valid for minor mergers, actually underestimates the merger time scale for them. This result is in approximate agreement with Colpi et al. (1999) who found that the friction timescale for $m_{\text {sat }} / m_{\text {pri }} \approx 0.02$ is underestimated by a factor of 2 if equation (11) is used. On the other side, our result points out that equation (1) significantly overestimates the dynamical friction time scales for major mergers. Despite the fact that the formula is not expected to be applicable to major mergers, it is still widely used for major mergers in the literature. Our findings do not agree with Navarro et al. (1995) who advocate a good agreement between their simulation result and equation (1) for minor mergers with mass ratios less than 0.5. In the next section, we will use our simulation data to improve the description for the merger timescale in the hierarchical clustering scenario.

\section{Fitting formula for the merger timescale in cosmological context}

First, because $r_{\mathrm{c}} \approx r_{\text {vir }}$ we rewrite the formula of $T_{\text {Chandra }}$ as

$$
T_{\text {Chandra }}=\frac{1}{2} \frac{f(\epsilon)}{C} \frac{m_{\mathrm{pri}}}{m_{\mathrm{sat}}} \frac{1}{\ln \Lambda} \frac{r_{\mathrm{c}}}{V_{\mathrm{c}}}
$$

where $r_{\mathrm{c}} / V_{\mathrm{c}} \propto 1 / \sqrt{G \rho}$ and $\rho$ is the mean mass density of the halo at that redshift. Thus $r_{\mathrm{c}} / V_{\mathrm{c}}$ is proportional to the age of the Universe at the 
epoch being considered 1 , independent of primary and/or satellite halo masses. Consequently, the mass dependence of $T_{\text {Chandra }}$ is solely accounted for by the mass ratio between satellite and primary halo, and its circularity dependence is included by the function $f(\epsilon)$. It is suggesting to isolate those two dependencies to find the cause of the discrepancies between the merger time scales derived from equation (11) and the simulation. Therefore, in the following section we will focus on the dependence of the merging time scales on the mass ratios. Subsequently, we will examine the circularity dependence in detail. Finally, these investigations will lead us to a new description of merger time scales in the cosmological context.

\subsection{Dependence on the mass ratio and Coulomb logarithm}

The strong dependence of $T_{\text {simu }} / T_{\text {Chandra }}$ on the mass ratio $m_{\text {sat }} / m_{\text {pri }}$ shown in Figure 3 (the black solid line) indicates that the mass dependence of $T_{\text {Chandra }}$ as described by equation (1) is incorrect. Here, we first consider to revise the Coulomb logarithm. In fact, in the original derivation of the formula (see, Binney \& Tremaine 1987), the Coulomb logarithm should read as $\frac{1}{2} \ln \left(1+\Lambda^{2}\right)$. Only if the satellite mass is much smaller than the primary mass this expression can be written as $\ln \Lambda$. In the literature $\frac{1}{2} \ln \left(1+\Lambda^{2}\right)$ is simply used to include mergers that do not satisfy the condition $m_{\text {sat }} \ll m_{\text {pri }}$ (e.g. Somerville \& Primack 1999). But another version, namely $\ln \left(1+m_{\text {pri }} / m_{\text {sat }}\right)$, is even more widely used for the same purpose (e.g. Springel et al. 2001; Volonteri et al. 2003; Kang et al. 2005) despite the fact that there is no clear physical motivation for adopting it. Here we examine the mass dependence using these two alternative forms for the Coulomb logarithm.

The red dashed line and the green dotted line in Figure 3 show the mass dependence of $T_{\text {simu }} / T_{\text {Chandra }}$ for these two alternative forms of the Coulomb logarithm. For mass ratios less than 0.1 the two curves are quite similar to that of $\ln \Lambda$ (solid line). But, for mass ratios $\sim 1$ they display substantial differences. The mass dependence becomes significantly smaller for these two forms, especially for the form $\ln \left(1+m_{\text {pri }} / m_{\text {sat }}\right)$,

\footnotetext{
${ }^{1} r_{c}$ is about $r_{\text {vir }}$ but there is scatter, so the statement is valid approximately
}

however, it does not disappear completely.

As a trial to improve the description for the mass dependence, we replace $r_{\mathrm{c}}$ in equation (11) by $r_{\text {vir }}$ for the two forms of the Coulomb logarithm mentioned above. Figures 4 and 5 show $T_{\text {simu }} / T_{\text {Chandra }}$ for $\frac{1}{2} \ln \left(1+\Lambda^{2}\right)$ and $\ln (1+$ $\left.m_{\text {pri }} / m_{\text {sat }}\right)$, respectively. The plot based on either of the two forms does not differ much. Here, we want to emphasize two points. First, the scatter in the plots is much smaller than in Figure 2. Second, the value of $T_{\text {Chandra }}$ is systematically smaller than that of $T_{\text {simu }}$. Although the scatter is smaller, it nevertheless will provide some deeper insight to examine if the scatter depends on the mass ratio. In analogy to Figure [3, we plot in the Figures [6] and 7 the median value of $T_{\text {simu }} / T_{\text {Chandra }}$ as a function of the mass ratio, for $\frac{1}{2} \ln \left(1+\Lambda^{2}\right)$ and $\ln \left(1+m_{\text {pri }} / m_{\text {sat }}\right)$, respectively. While there is a moderate dependence on the mass ratio when the form $1 / 2 \ln \left[1+\left(m_{\text {pri }} / m_{\text {sat }}\right)^{2}\right]$ is used, it is very interesting to recognize that the dependence of $T_{\text {simu }} / T_{\text {Chandra }}$ on the mass ratio for $\ln \left(1+m_{\text {pri }} / m_{\text {sat }}\right)$ is strongly reduced. This implies that the mass dependence of the merger time scale can be well represented by the form $\ln \left(1+m_{\text {pri }} / m_{\text {sat }}\right)$, though many previous works using this form actually systematically underestimate the merger time scale or overestimate the merger rate by a factor 2 .

In the following discussion, we will always use the form $\ln \left(1+m_{\text {pri }} / m_{\text {sat }}\right)$ for the Coulomb logarithm. We prefer to use this form with $r_{\mathrm{c}}$ replaced by $r_{\text {vir }}$ as this gives a much tighter correlation between $T_{\text {Chandra }}$ and $T_{\text {simu }}$ and can effectively absorb the dependence on the mass ratio. Moreover, in many practical applications, it is usually easier to use $r_{\text {vir }}$ than to use $r_{\mathrm{c}}$.

\subsection{Dependence on circularity and the re- vised form of $f(\epsilon)$}

Now we check the dependence of the merger time on the initial circularity parameter $\epsilon$. This parameter is determined from the velocity and position of a satellite when it first crosses the virial radius of the primary halo. As in the literature, we assume that the halo is an isothermal sphere when determining the circularity. In Figure 8 (upper line), we show the median value of $T_{\text {simu }} / T_{\text {Chandra }}$ as a function of circularity, where we have used $\ln \left(1+m_{\text {pri }} / m_{\text {sat }}\right)$ for the Coulomb logarithm and 
$f(\epsilon)=\epsilon^{0.78}$ when we calculate $T_{\text {Chandra }}$. The figure shows that the satellites on very eccentric orbits tend to merge in a much longer timescale compared to the theoretical prediction. If we still use an exponential form to represent $f(\epsilon)=\epsilon^{\alpha}$, the exponent $\alpha$ should be smaller than the widely used value 0.78 advocated by Lacev \& Cole (1993).

Here we explore the form of $f(\epsilon)$ as a function of initial circularity $\epsilon$. Substituting the merging time in equation (11) with what we measure in the simulation, $r_{\mathrm{c}}$ with $r_{\mathrm{vir}}$, and the Coulomb logarithm with $\ln \left(1+m_{\text {pri }} / m_{\text {sat }}\right)$, we obtain the values of $f(\epsilon)$ for each merged satellite. Subsequently, we pick the median value of $f(\epsilon)$ in each circularity bin in our merged satellite sample. Computing the median value, however, demands some caution. Because there is considerable scatter in $T_{\text {simu }}$ even for the same circularity and the same mass ratio (which owes to fact that internal structure and merger history of the primary halo may introduce some scatter into the merger time scale), there may exist a selection (or incompleteness) bias against those satellites of long $T_{\text {merger }}$. Those mergers would happen after our fifth last snapshot and thus be missed in our study. This effect gets more severe at larger $\epsilon$, because the merger times become systematically longer on more circular orbits. As a result, only those mergers with smaller $T_{\text {merger }}$ (for the same $\epsilon$ ) are selected into the merger sample, which will artificially lower the estimate of $f(\epsilon)$ for large $\epsilon$. In order to avoid such selection bias for the determination of $f(\epsilon)$, we construct a complete merger sample of primary halos and satellites at the first 14 snapshots (redshift $1.55 \sim 2.0$ ) with mass ratio greater than 0.1 (152 pairs). In this sample, all these satellites but 2 are found to have merged with the central galaxies of the primary halos before the fifth last snapshot. Therefore, our sample is complete for measuring $f(\epsilon)$ except for the bin at $\epsilon=0.50$ where the completeness is $98 \%$ and the bin at $\epsilon=0.70$ with the completeness of $97 \%$.

In Figure 9 we present our estimate of $f(\epsilon)$ from this complete sample. We first fit the data with $f(\epsilon)=a \epsilon^{\alpha}$ and find the best fitting values $a=1.48$ and $\alpha=0.27$. The fitting curve is displayed by the dashed line. If we use all mergers identified instead of the complete sample, the function $f(\epsilon)$ would be underestimated at larger $\epsilon$ as shown by the triangles in the figure, which in turn would lead to an even smaller $\alpha$. However, the degree of the underestimation becomes less serious, since the dependence on $\epsilon$ as shown by the complete sample is much weaker than the original function $f(\epsilon)=\epsilon^{0.78}$. To check if the circularity dependence in Figure 8 is fully accounted for by this fitting formula, we plot the median value of $T_{\text {simu }} / T_{\text {fit }}$ as a function of the circularity parameter (dotted line in Figure 8). Compared with the dashed line $\left(f(\epsilon)=\epsilon^{0.78}\right)$ the dependence on $\epsilon$ is strongly reduced. However, we note that the new time ratio is still a little higher for the smallest circularity bin. This can be contributed to the artificial effect of the pure exponential fitting form which falsely results in a vanishing merger timescale for $\epsilon=0$. If we consider two merging halos with equal masses and assume they will merge within a free fall time scale $r_{\mathrm{vir}} / V_{\mathrm{c}}$, equation (4) gives $f(\epsilon)=0.60$. Therefore, to avoid the artificial effect at $\epsilon=0$ due to the pure exponential form and to reduce the somewhat too high time ratio in the smallest circularity bin we fit our simulation data with $f(\epsilon)=a \epsilon^{\alpha}+0.60$. The best fitting results are $a=0.94$ and $\alpha=0.60$. The solid line in Figure 9 shows the best fitting curve which matches the data very well. The solid line in Figure 8 demonstrates that the $T_{\text {simu }} / T_{\text {fit }}$ in the first circularity bin has moderately decreased, now approaching to a value of 1 .

The exponent $\alpha$ we find here is much smaller than the widely used value $\alpha=0.78$. At this point it is worth recalling that $\alpha=0.78$ was obtained by Lacey \& Cole (1993) analytically for the case where a rigid satellite falls into an isothermal sphere. The fact that our $f(\epsilon)$ always exceeds $\epsilon^{0.78}$ can be interpreted as an indication for the mass loss of satellites in the simulation (cf., Colpi et al. 1999). Satellites on radial orbits lose their mass much faster than those on circular orbits, which implies that satellites on the radial orbits show relatively prolonged merging time scales compared to satellites on circular orbits. Therefore, one expects higher values for $f(\epsilon)$ or equivalently lower values for $\alpha$ for small $\epsilon$. In a future paper(Faltenbacher et al. 2007, in preparation), we will explore this qualitative explanation using an analytical model similar to those of Zentner \& Bullock (2003) and Zentner et al. (2005) (see also, Bullock et al. 2000; Taylor \& Babul 2001, 2004). 


\subsection{Taking into account both the mass and circularity dependencies}

Combining our results on the mass and circularity dependencies, we write the merger time scale as

$$
T_{\mathrm{fit}}=\frac{0.94 \epsilon^{0.60}+0.60}{2 C} \frac{m_{\mathrm{pri}}}{m_{\mathrm{sat}}} \frac{1}{\ln \left[1+\left(\frac{m_{\mathrm{pri}}}{m_{\mathrm{sat}}}\right)\right]} \frac{r_{\mathrm{vir}}}{V_{\mathrm{c}}} .
$$

In Figure 10 this equation is compared with the merger time scale of all mergers measured in the simulation. Remarkably, the scatter in the plot is much smaller than that in Figures 4 and 5 , indicating that $T_{\text {fit }}$ describes the merger timescale much better than equation (1). To assess the scatter in more detail Figure 11 displays the distribution of $T_{\text {simu }} / T_{\text {fit }}$. The solid histogram shows the distribution based on the early complete merger sample as described in 4.2 and the dotted histogram gives the distribution for all mergers identified in our simulation.

The distribution for the sample of all mergers is shifted towards the left relative to the complete sample. This is caused by the lack of long time mergers among recently infalling satellites in the sample of all mergers. These long time mergers would be included in the sample if the simulation were evolved beyond the present time $z=0$. This also leads to the trend of the data points to lie slightly above the solid diagonal in Figure 10.

The distribution of $x=T_{\text {simu }} / T_{\text {fit }}$ is well fitted by the log-normal distribution

$$
p(\ln x) d \ln x=\frac{1}{\sqrt{2 \pi} \sigma} \exp \left[-\frac{(\ln x)^{2}}{2 \sigma^{2}}\right] d \ln x
$$

with $\sigma=0.4$ (the smooth solid line in Figure 11). This distribution function combined with the fitting function (5) provides a description for the merger time in a statistical sample.

From Figure 8 we have learned that the circularity dependence is accounted for by equation (5). Now, we examine the mass dependence when equation (5) is applied. Therefore, we plot the median value of $T_{\text {simu }} / T_{\text {fit }}$ as a function of the mass ratio in Figure 7 (solid line). Strikingly, we find no dependence on the mass ratio which indicates that the dependencies of the merger time on mass and circularity are completely reproduced by equation (5).

\subsection{Distribution of circularity}

Once the distribution of circularity is known for a population of infalling satellites, one can determine how many of the satellites will merge into central galaxies at a certain epoch in a statistical way by using equations (5) and (6). Various authors (e.g., Tormen 1997; Zentner et al. 2005; Khochfar \& Burkert 2006) have studied this distribution, with similar conclusions that orbits with intermediate $\epsilon$ are common while those at both ends $(\epsilon \approx 0$ or 1$)$ are rare. Figure 12 shows our result for all resolved satellite halos, with an average value of $\epsilon$ about 0.51 , which is consistent with $0.53 \pm 0.23$ in Tormen (1997). The distribution can be well described by

$$
p(\epsilon) d \epsilon=2.77 \epsilon^{1.19}(1.55-\epsilon)^{2.99} d \epsilon
$$

which is shown as the solid line in Figure 12 The circularity distribution is independent of the mass ratio of the primary halo and the satellite, as shown by Figure 13.

It is worth noting that in semi-analytical models, the circularity parameter was often randomly drawn from a uniform distribution between 0 and 1 (e.g., Kauffmann et al. 1999; Somerville \& Primack 1999). According to our findings such an approach biases the estimate for the input dynamical friction timescales.

\section{Conclusions and discussion}

In this paper, we have analysed galaxy mergers in a $\mathrm{SPH} / \mathrm{N}$-body simulation and compared the merger time scale with the theoretical prediction based on the Chandrasekhar formula. We have obtained the following main conclusions.

- In contrast with Navarro et al. (1995), we find that the widely used equation (1) with the satellite's total mass at its first crossing of the host virial radius taken for $m_{\text {sat }}$, systematically underestimates the merger timescale for minor mergers and overestimates it for major mergers;

- We show that the two alternative forms $\ln \left(1+m_{\text {pri }} / m_{\text {sat }}\right)$ and $1 / 2 \ln \left[1+\left(m_{\text {pri }} / m_{\text {sat }}\right)^{2}\right]$ for the Coulomb logarithm, which also are widely used in literature, account for the 
mass dependence of merger timescale successfully. However, both of them underestimate the merger time scale by a factor 2 if the satellite's total mass at its first crossing of the host virial radius is used for $m_{\text {sat }}$. Of these two forms, the former does slightly better in accounting for the mass dependence;

- With $\ln \left(1+m_{\text {pri }} / m_{\text {sat }}\right)$ taken for the Coulomb logarithm, we find the dependence on circularity parameter $\epsilon$ is much weaker than $\epsilon^{0.78}$, and can be accurately represented by $0.94 \epsilon^{0.60}+0.60$

- Combining our findings on the mass and circularity dependencies, we present an accurate fitting formula (eq 5) for the merger timescale. Together with the distribution functions (eqs [6 and 7), one can use this equation to predict for mergers of galaxies in LCDM models.

Our results do not necessarily mean that Chandrasekar's theory is not applicable for mergers of galaxies. Instead our results do indicate that many previous applications of this theory led to incorrect results because some simplified assumptions were adopted. We believe that the mass loss of satellites and the steep density gradient of host halos are two of the key reasons that make the problem complicated. In a future paper, we will investigate if our simulation results can be reproduced with the Chandrasekhar theory by properly taking into account of these two factors.

In the following we will discuss how potential shortcomings in the treatment of the baryonic physics at the core of the primary halo may affect our results. It is well known that current hydrodynamic simulations suffer from the so called 'overcooling' problem, i.e. the gas at the core of massive dark matter halos cools too rapidly resulting in too massive central galaxies compared to observations (e.g., Borgani et al. 2004; Saro et al. 2006; Naab et al. 2007). In turn, adiabatic contraction (e.g., Gnedin et al. 2004) may also change the dark matter properties at the central parts of the halo in an unphysical manner.

However, we think that this process does not substantially alter our results for two reasons: (1) With exception of very radial orbits, which are rare, the satellite galaxies spend most of their time during the merging process at the outer parts of the primary halo where dynamical friction is moderate. If, however, the satellite is once migrated towards the central parts of the primary halo dynamical friction becomes very efficient and the remaining lifetime of the satellite galaxy is short. Consequently, the merger time scale is set by the conditions at the outer parts of the primary halo (c.f., Navarro et al. 1995). (2) The findings of Springel et al. (2001) and Kang et al. (2005) are in qualitative agreement with our results. Since both of these studies are based on pure N-body simulations they obviously do not suffer from the overcooling problem. This is an further indication that our results are accurate and are not affected by the potential shortcomings in the treatment of the baryonic physics in the simulations. These arguments are supported by the left panel of Figure 14. which shows that there is no dependence of $T_{\text {simu }} / T_{\text {fit }}$ on $m_{\text {stellar }} / m_{\text {pri }}$, the ratio of the central galaxy's stellar mass to the dark matter mass of the surrounding primary halo.

We have also checked if our result is affected by the growth of the primary halo during the merger course. The middle panel of Figure 14 shows the ratio $T_{\text {simu }} / T_{\text {fit }}$ as a function of the mass growth rate of the primary halo, which is defined as the ratio of its dark matter mass at the time of merger to its mass at the time of the first crossing. The result indicates that the merger time scale is not affect by the growth of the primary halo. A possible explanation is that the internal density structure does not change significantly during this course. Of course, violent major mergers may change the internal structures and bring about large fluctuations in the merger time.

To keep the fitting formula simple to use, we prefer not to include the dependence on the energy of the satellite's orbit, that is, on $r_{c}$. We have examined this dependence in the right panel of Figure 14, which shows there is a weak dependence on $r_{c} / r_{\text {vir }}$. We can include this dependence in our fitting formula by replacing $r_{\text {vir }}$ with $\sqrt{r_{\text {vir }} r_{c}}$. Thus, the fitting formula with the $r_{c}$-dependence included reads as

$$
T_{\text {fit }}=\frac{0.90 \epsilon^{0.47}+0.60}{2 C} \frac{m_{\text {pri }}}{m_{\text {sat }}} \frac{1}{\ln \left[1+\left(\frac{m_{\text {pri }}}{m_{\mathrm{sat}}}\right)\right]} \frac{\sqrt{r_{\mathrm{vir}} r_{c}}}{V_{\mathrm{c}}},
$$


and Figure14 shows that the $r_{c}$ dependence is fully accommodated by this simple heuristic correction. We have checked the dependence on the mass ratio $m_{\text {pri }} / m_{\text {sat }}$ as well as the scatter in $T_{\text {simu }} / T_{\text {fit }}$, and found that they are nearly the same as when equation (5) is used. The better performance of equation (8) is achieved at the expense of computing the energy of the individual satellite orbits. Since the accuracy of the fitting formula is improved only slightly by including the $r_{c}$ dependence compared with the scatter in $T_{\text {simu }} / T_{\text {fit }}$, the simpler formula (5) is preferred for most applications.

As concluding remark, we once again focus our attention on equation (5) which can be considered as the distillate of our analysis. This fitting formula allows to predict the merger timescale $T_{\text {fit }}$ for the two central galaxies within a satellite and a primary halo. The merger timescale for the satellite galaxy is defined as the time which elapses between its first crossing of the primary's virial radius and its final coalescence with the central galaxy. The computation of accurate merger timescales is a crucial ingredient for semianalytical modeling of galaxy formation.

Equation (5) requires two input values: $m_{\text {pri }} / m_{\text {sat }}$, the mass ratio of primary and satellite halo (before they start merging) and $\epsilon=J / J(E)$, the satellites initial circularity which is defined as the ratio of the satellites actual angular momentum $J$ and the angular of a circular orbit with the same energy $J(E) . r_{\text {vir }}$ is the virial radius of the primary halo just before the satellite merges with it. The factors $C$ and $r_{\text {vir }} / V_{c}$ are constants and do not depend on the specific constellation. If $\epsilon$ is not known it can be randomly drawn from the distribution provided by equation (7) which we have derived directly from the simulation data, see Figure 12. This random process can be applied for arbitrary mass ratios $\left(m_{\text {pri }} / m_{\text {sat }}\right)$ since the distributions of $\epsilon$ are nearly independent of mass as shown in Figure 13.

Finally, it remains to mention that due to stochastic processes during a merger event, like close encounters with other substructures or the occurrence of multiple mergers at the same time, there arises substantial scatter among the merger time scales with equivalent initial conditions. This can be taken into account if the values for $T_{\text {fit }}$ obtained from equation (5) are spread according to the log-normal distribution given in equation (6) which is also displayed in Figure 11. With the fitting formula (5) we provide a robust estimate of the merger timescale pivotal for all kinds of analytical modeling of galaxy evolution within dark matter halos.

After we submitted our paper both to the journal and to the astro-ph electronic library, an independent work by Boylan-Kolchin et al. (2007) on the same subject had appeared on the electronic library. Their paper is qualitatively consistent with ours in that the time scale given by equation (11) is underestimated. But quantitatively, their results are rather different from ours. Both the dependencies on the mass ratio and the circularity parameters are much stronger in their paper. In particular, the strong dependence on the circularity they found, which is even stronger than $\epsilon^{0.78}$ at $\epsilon=0.5-1$, is not consistent with our data. The dependence on the mass ratio is also stronger than ours. This discrepancy may mainly come from the difference between the simulations: they present a series of pure N-body simulations of two halo mergers, whereas our results are based on a cosmological hydro/N-body simulation with star formation. While our fitting formula is accurate for mergers in the cosmological frame, future work is still needed to find out the specific causes of this discrepancy.

We would like to thank Volker Springel for providing the Gadget Code with star formation and Liang Gao for his help with using the code. This work is supported by NSFC (10533030, 0742961001, 0742951001), by Shanghai Key Projects in Basic research (No. 04JC14079 and 05XD14019), and by the Knowledge Innovation Program of the Chinese Academy of Sciences, Grant No. KJCX2-YW-T05. AF is supported by the CAS Research Fellowship for International Young Researchers. The simulation was performed at the Shanghai Supercomputer Center.

\section{REFERENCES}

Baugh, C. M. 2006, Reports of Progress in Physics, 69, 3101

Benson, A. J. 2005, MNRAS, 358, 551 
Binney, J., \& Tremaine, S. 1987, Princeton, NJ, Princeton University Press, 1987, 747 p.,

Bryan, G. L., \& Norman, M. L. 1998, ApJ, 495, 80

Borgani, S., et al. 2004, MNRAS, 348, 1078

Boylan-Kolchin, M., Ma, C.-P., \& Quataert, E. 2007, ArXiv e-prints, 707, arXiv:0707.2960

Bullock, J. S., Kolatt, T. S., Sigad, Y., Somerville, R. S., Kravtsov, A. V., Klypin, A. A., Primack, J. R., \& Dekel, A. 2001, MNRAS, 321, 559

Bullock, J. S., Kravtsov, A. V., \& Weinberg, D. H. 2000, ApJ, 539, 517

Chandrasekhar, S. 1943, ApJ, 97, 255

Cole, S., Lacey, C. G., Baugh, C. M., \& Frenk, C. S. 2000, MNRAS, 319, 168

Colpi, M., Mayer, L., \& Governato, F. 1999, ApJ, 525,720

Conroy, C., Ho, S., \& White, M. 2007, ArXiv eprints, 706, arXiv:0706.0520

Cora, S. A., Muzzio, J. C., \& Vergne, M. M. 1997, MNRAS, 289, 253

Diemand, J., Moore, B., \& Stadel, J. 2004, MNRAS, 352, 535

in preparation

Fellhauer, M., \& Lin, D. N. C. 2007, MNRAS, 375, 604

Fujii, M., Funato, Y., \& Makino, J. 2006, PASJ, 58,743

Gao, L., White, S. D. M., Jenkins, A., Stoehr, F., \& Springel, V. 2004, MNRAS, 355, 819

Gill, S. P. D., Knebe, A., \& Gibson, B. K. 2005, MNRAS, 356, 1327

Gnedin, O. Y., Kravtsov, A. V., Klypin, A. A., \& Nagai, D. 2004, ApJ, 616, 16

Gnedin, O. Y. 2003, ApJ, 582, 141

Gonzalez, A. H., Zaritsky, D., \& Zabludoff, A. I. 2007, ApJ, 666, 147
Hashimoto, Y., Funato, Y., \& Makino, J. 2003, ApJ, 582, 196

Hatton, S., Devriendt, J. E. G., Ninin, S., Bouchet, F. R., Guiderdoni, B., \& Vibert, D. 2003, MNRAS, 343, 75

Jiang, I.-G., \& Binney, J. 2000, MNRAS, 314, 468

Jing, Y. P., Zhang, P., Lin, W. P., Gao, L., \& Springel, V. 2006, ApJ, 640, L119

Kang, X., Jing, Y. P., Mo, H. J., Börner, G. 2005, ApJ, 631, 21

Kauffmann, G., Colberg, J. M., Diaferio, A., \& White, S. D. M. 1999, MNRAS, 303, 188

Khochfar, S., \& Burkert, A. 2006, A\&A, 445, 403

Khochfar, S., \& Ostriker, J. P. 2007, ArXiv eprints, 704, arXiv:0704.2418

King, I. 1962, AJ, 67, 471

Lacey, C., \& Cole, S. 1993, MNRAS, 262, 627

Lin, L., et al. 2004, ApJ, 617, L9

Lin, Y.-T., Mohr, J. J., \& Stanford, S. A. 2003, ApJ, 591, 749

Maller, A. H., Katz, N., Kereš, D., Davé, R., \& Weinberg, D. H. 2006, ApJ, 647, 763

Menci, N., Cavaliere, A., Fontana, A., Giallongo, E., \& Poli, F. 2002, ApJ, 575, 18

Menci, N., Fontana, A., Giallongo, E., Grazian, A., \& Salimbeni, S. 2006, ApJ, 647, 753

Monaco, P., Fontanot, F., \& Taffoni, G. 2007, MNRAS, 375, 1189

Monaco, P., Salucci, P., \& Danese, L. 2000, MNRAS, 311, 279

Moore, B., Katz, N., Lake, G., Dressler, A., \& Oemler, A. 1996, Nature, 379, 613

Nagashima, M., Yoshii, Y., Totani, T., \& Gouda, N. 2002, ApJ, 578, 675

Naab, T., Johansson, P. H., Ostriker, J. P., \& Efstathiou, G. 2007, ApJ, 658, 710

Navarro, J. F., Frenk, C. S., \& White, S. D. M. 1995, MNRAS, 275, 56 
Ostriker, J. P., \& Turner, E. L. 1979, ApJ, 234, 785

Saro, A., Borgani, S., Tornatore, L., Dolag, K., Murante, G., Biviano, A., Calura, F., \& Charlot, S. 2006, MNRAS, 373, 397

Shaw, L. D., Weller, J., Ostriker, J. P., \& Bode, P. 2007, ApJ, 659, 1082

Somerville, R. S., \& Primack, J. R. 1999, MNRAS, 310,1087

Springel, V., Yoshida, N., \& White, S. D. M. 2001, New Astronomy, 6, 79

Springel, V. 2005, MNRAS, 364, 1105

Springel, V., \& Hernquist, L. 2003, MNRAS, 339, 312

Springel, V., White, S. D. M., Tormen, G., \& Kauffmann, G. 2001, MNRAS, 328, 726

Taylor, J. E., \& Babul, A. 2004, MNRAS, 348, 811

Taylor, J. E., \& Babul, A. 2001, ApJ, 559, 716

Tormen, G. 1997, MNRAS, 290, 411

Tormen, G., Diaferio, A., \& Syer, D. 1998, MNRAS, 299, 728

van den Bosch, F. C., Lewis, G. F., Lake, G., \& Stadel, J. 1999, ApJ, 515, 50

Volonteri, M., Haardt, F., \& Madau, P. 2003, ApJ, 582,559

von Hoerner, S. 1957, ApJ, 125, 451

White, M., Zheng, Z., Brown, M. J. I., Dey, A., \& Jannuzi, B. T. 2007, ApJ, 655, L69

White, S. D. M. 1976, MNRAS, 174, 467

Zaritsky, D., \& White, S. D. M. 1988, MNRAS, 235,289

Zentner, A. R., Berlind, A. A., Bullock, J. S., Kravtsov, A. V., \& Wechsler, R. H. 2005, ApJ, 624,505

Zentner, A. R., \& Bullock, J. S. 2003, ApJ, 598, 49

Zheng, Z., Coil, A. L., \& Zehavi, I. 2007, ApJ, 667,760

This 2-column preprint was prepared with the AAS IATEX
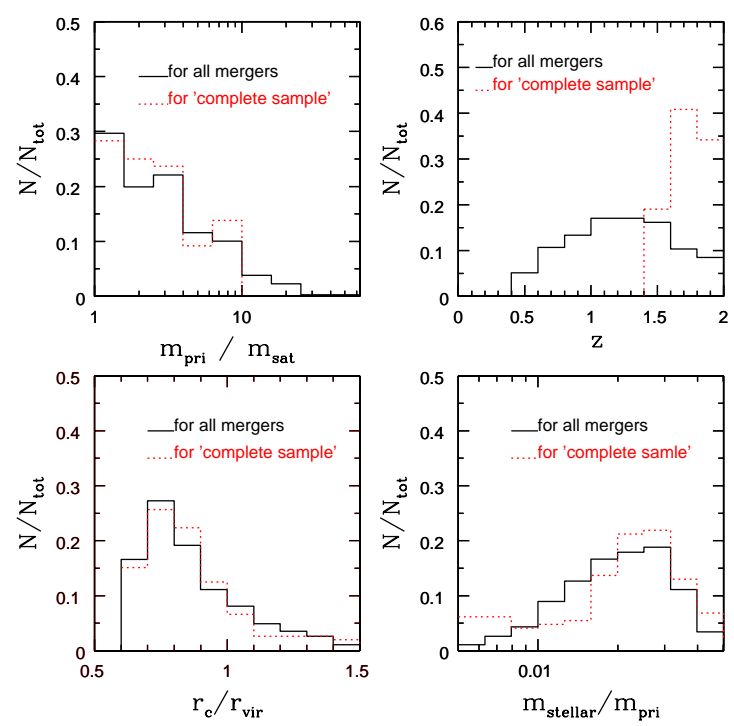

Fig. 1. - Basic characteristics of all mergers (solid histograms) and the mergers in the complete sample (dashed histograms). The panels from the top left to the bottom left clockwise show the distributions of the mass ratio of the primary halo to the satellite, the first crossing redshift, the mass ratio of the stellar mass of the central galaxy to the primary, and the ratio of $r_{c}$ to the virial radius $r_{\text {vir }}$ respectively. 


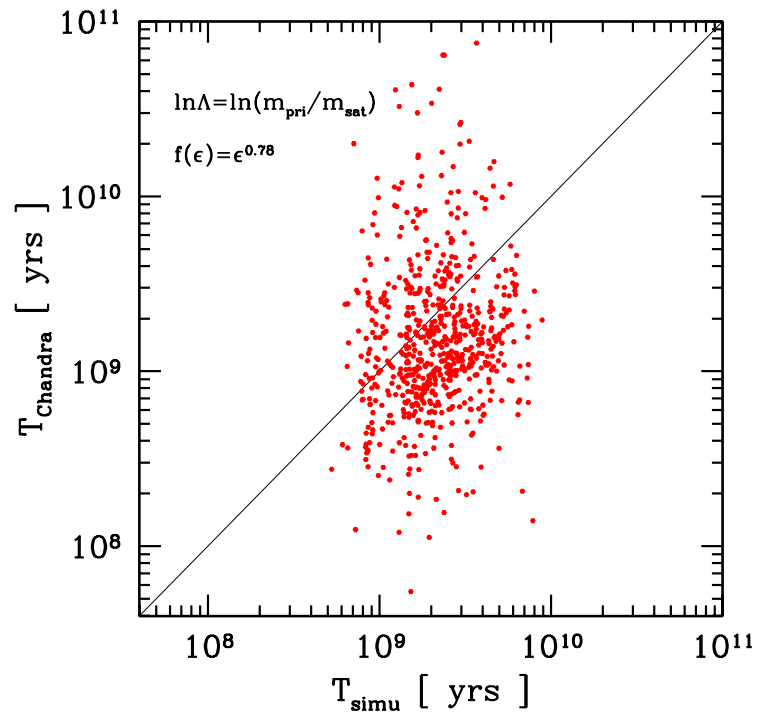

Fig. 2.- Comparison of the merger timescale $T_{\text {simu }}$ in the simulation with theoretical dynamical friction timescale $T_{\text {Chandra }}$ from equation (1). The solid line is $T_{\text {Chandra }}=T_{\text {simu }}$. The Coulomb logarithm is in the form $\ln \Lambda=\ln \left(m_{\mathrm{vir}} / m_{\mathrm{sat}}\right)$, and $f(\epsilon)=\epsilon^{0.78}$.

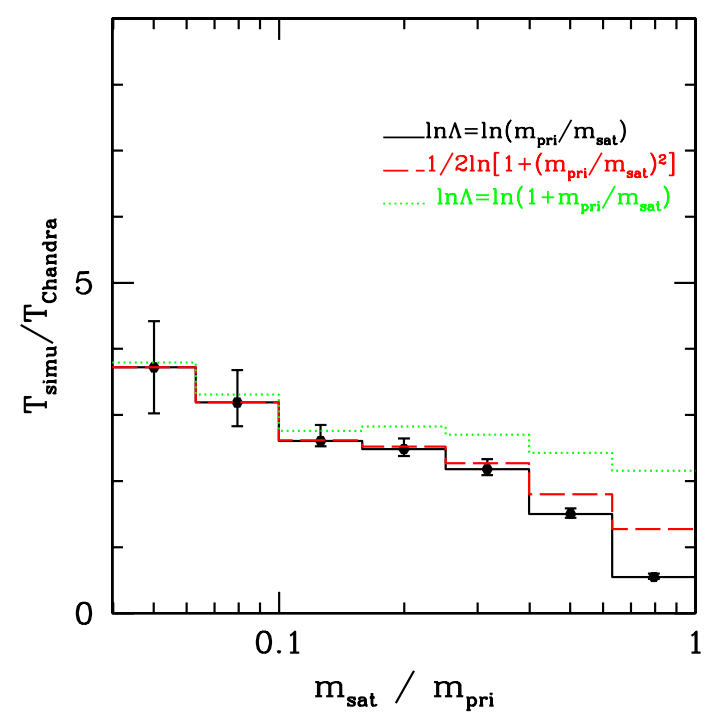

Fig. 3.- Mass dependence of the median value of $T_{\text {simu }} / T_{\text {Chandra }}$, when different forms are used for the Coulomb logarithm.

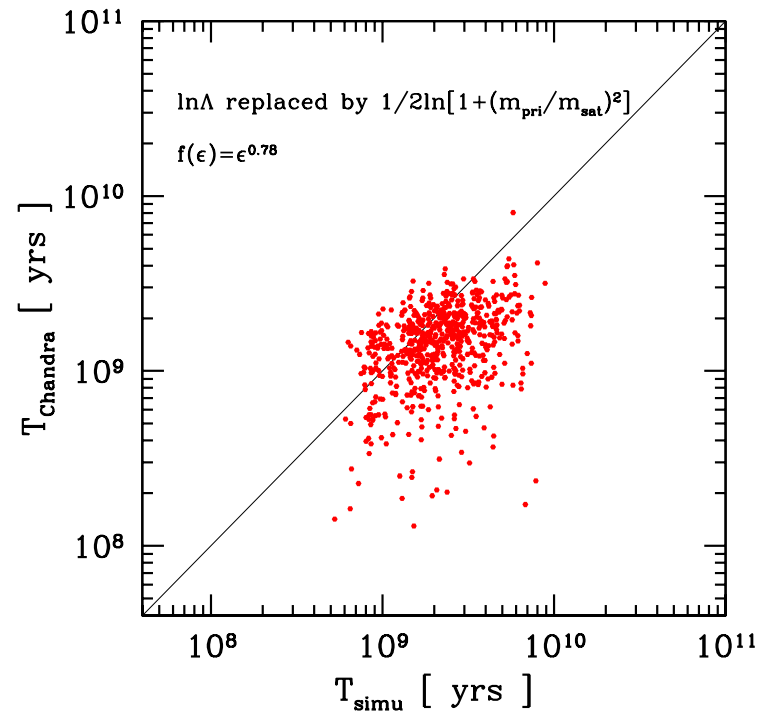

Fig. 4. - The same as Figure 1, but we replace $\ln \Lambda$ by $\frac{1}{2} \ln \left(1+\Lambda^{2}\right)$, while $\Lambda$ is unchanged, and replace $r_{\mathrm{c}}$ by $r_{\mathrm{vir}}$.

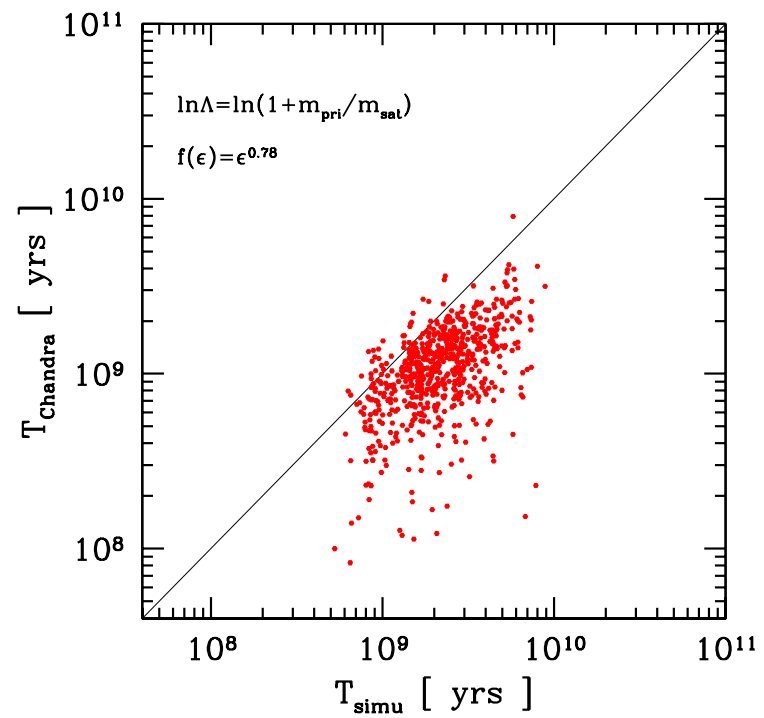

Fig. 5. - The same as Figure 1, but we replace $\ln \Lambda$ by $\ln (1+\Lambda)$, and replace $r_{\mathrm{c}}$ by $r_{\mathrm{vir}}$. 


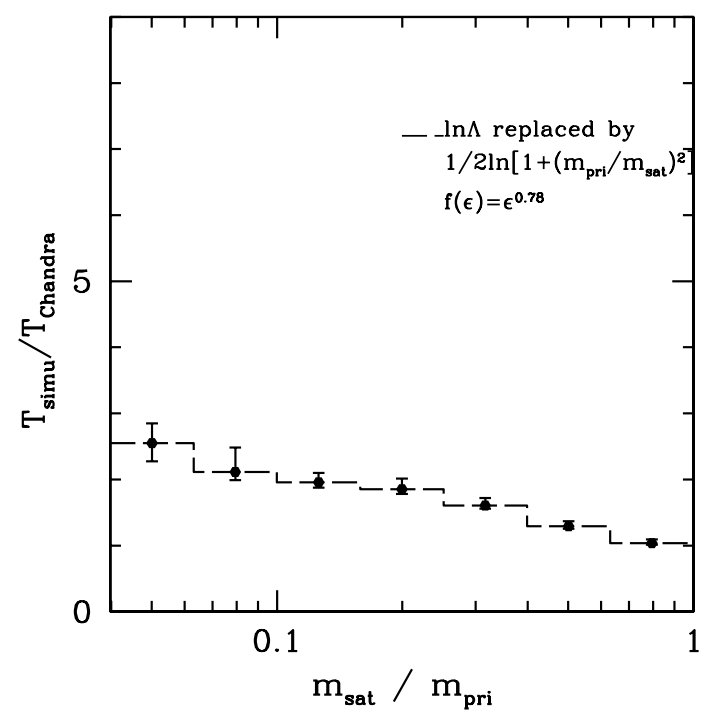

Fig. 6. - The ratio of $T_{\text {simu }}$ to $T_{\text {Chandra }}$ as a function of mass ratio for the merger points in Figure 4.

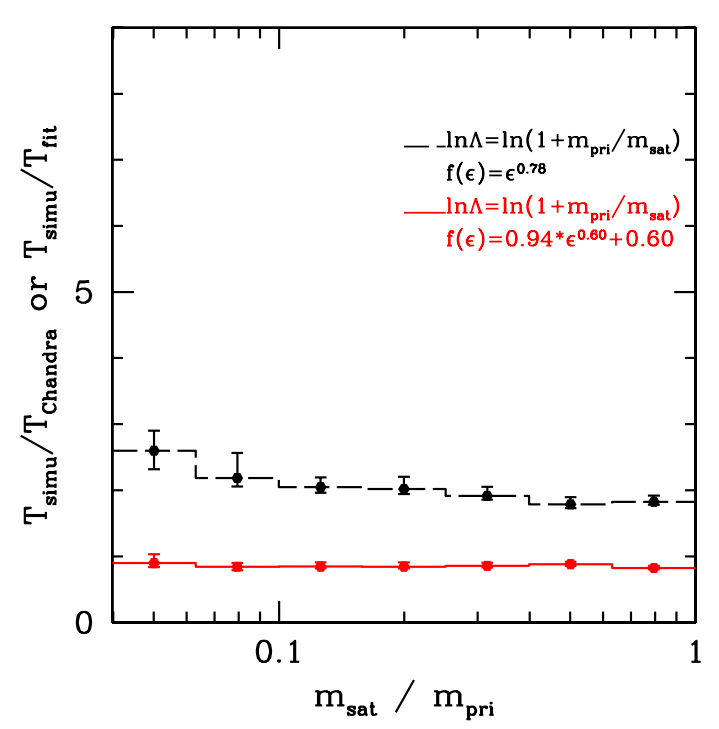

Fig. 7.- The ratio of $T_{\text {simu }}$ to $T_{\text {Chandra }}$ as a function of mass ratio for merger points in Figure 5 (the upper dashed line) and for those after applying equation (5) (the lower solid line).

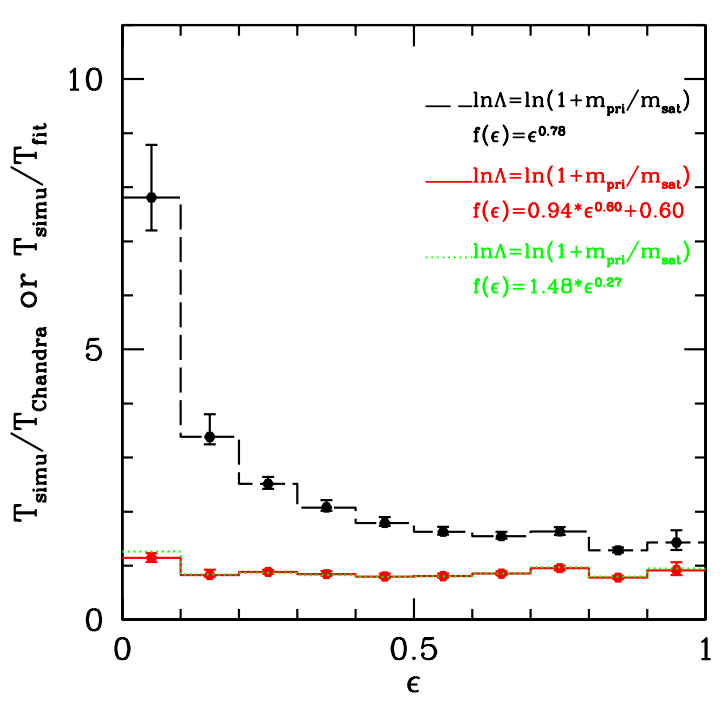

Fig. 8. - The ratio of $T_{\text {simu }}$ to $T_{\text {Chandra }}$ as a function of $\epsilon$ for merger points in Figure 5 (the upper dashed line) and for those after applying equation (5) (the lower solid line). The lower dotted line is for $f(\epsilon)=1.48 * \epsilon^{0.27}$ with equation (5).

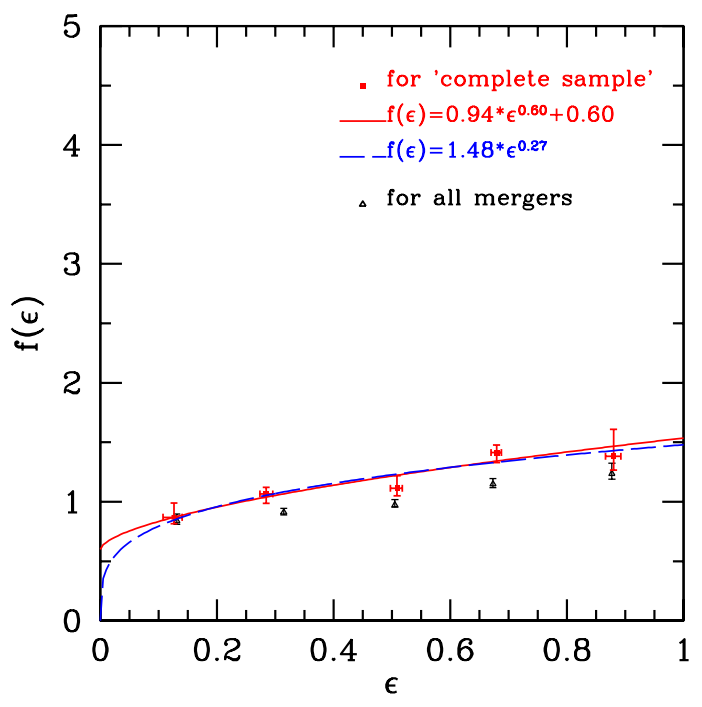

Fig. 9.- Fitting function of $f(\epsilon) . \quad f(\epsilon)=0.94 *$ $\epsilon^{0.60}+0.60$ is represented by the solid curve, while $f(\epsilon)=1.48 * \epsilon^{0.27}$ is denoted by the dashed line. The squares are from the complete sample of mergers, and the triangles are from all mergers. 


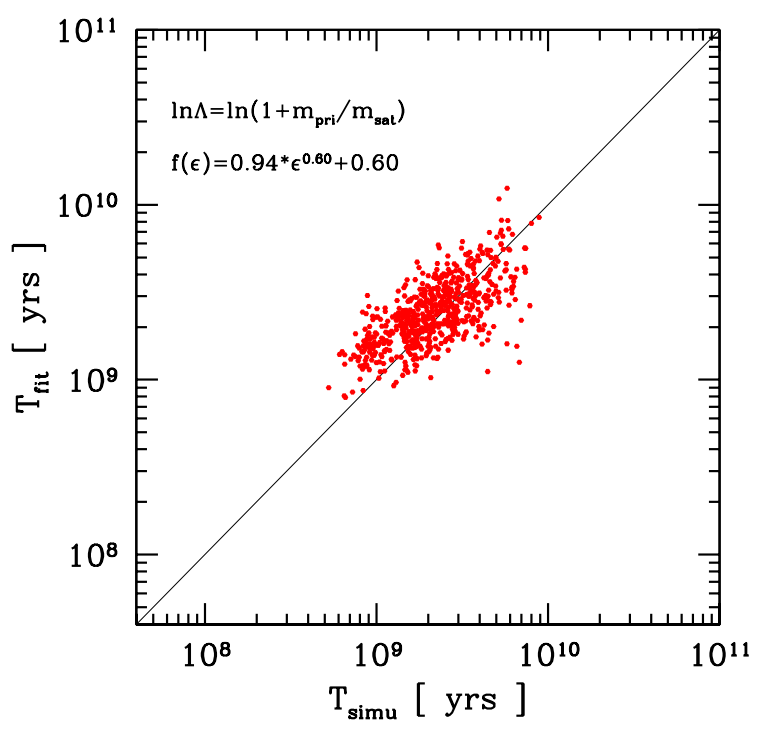

Fig. 10.- Comparison of the merger time scale from simulation with our fitted merger time scale (equation 5). The data points lie slightly above the solid line, because the sample missed a mall fraction of relatively long mergers.

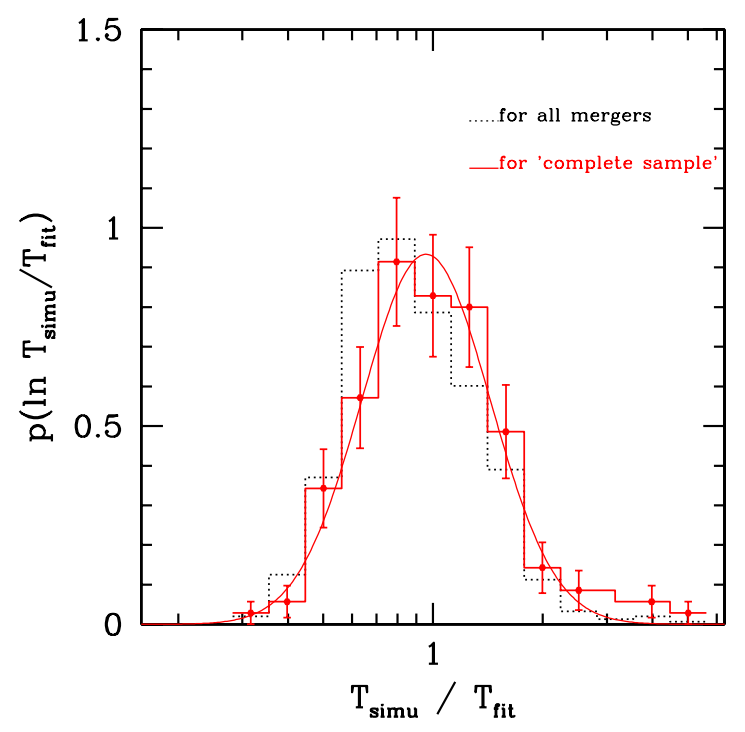

Fig. 11. - Distribution of $T_{\text {simu }} / T_{\text {fit }}$ for the whole merger sample(the dotted line) and for the complete sample(the solid line). The left shift of the whole merger sample is mainly due to its lack of those relatively long mergers.

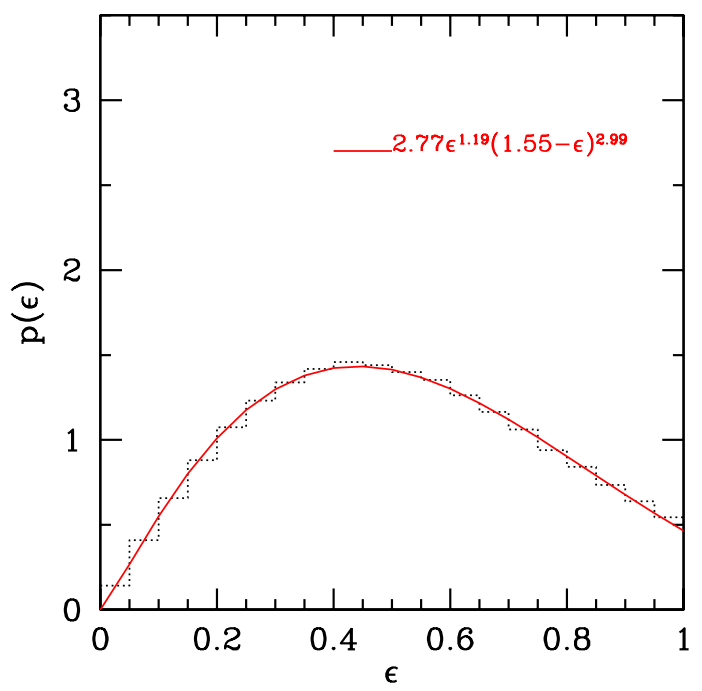

Fig. 12.- Distribution of circularity parameter $\epsilon$ for all resolved satellite halos having more than half their masses entering the primary halos.

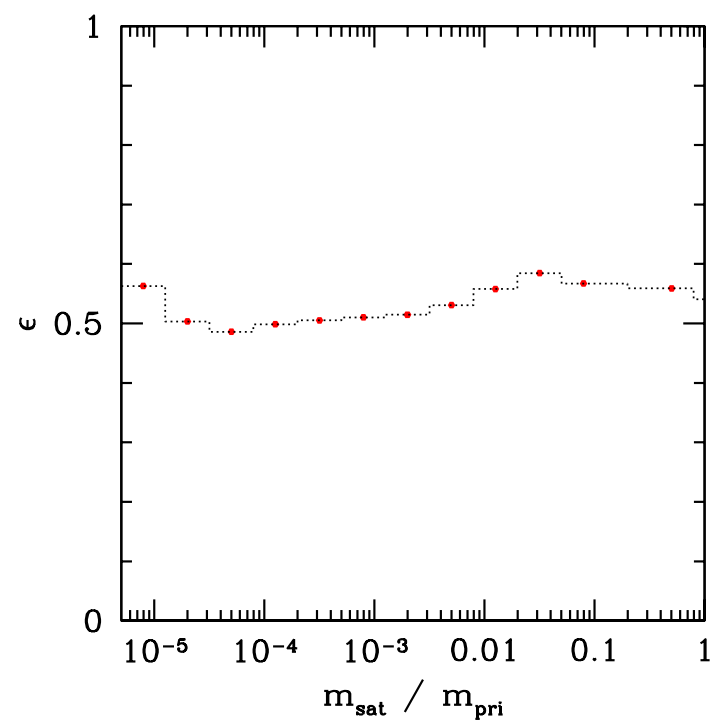

Fig. 13. - The mean circularity as a function of the mass ratio. 


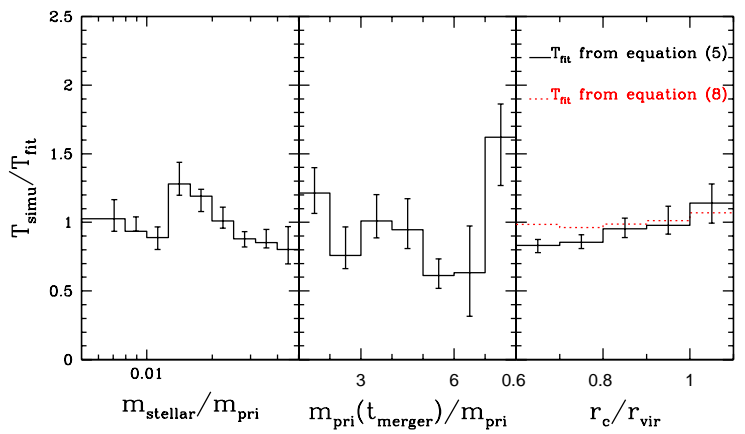

Fig. 14. - The ratio $T_{\text {simu }} / T_{\text {fit }}$ as a function of the mass ratio $m_{\text {stellar }} / m_{\text {pri }}$ (left), the growth rate of the primary halo (middle), and $r_{c} / r_{\text {vir }}$ (right) for the complete sample.

macros v5.2. 